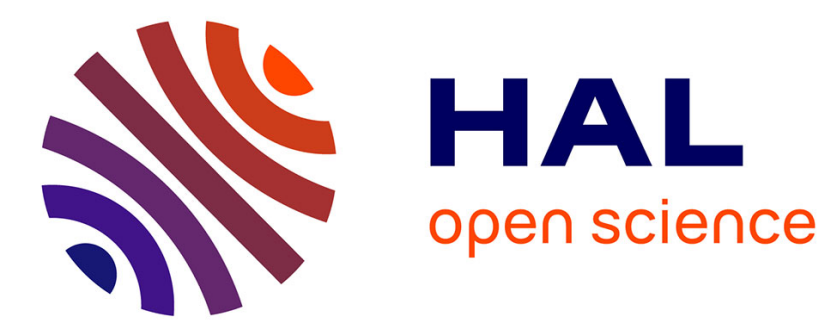

\title{
Relation entre l'activité fixatrice d'azote non symbiotique et quelques propriétés physicochimiques du sol
}

Manuel Tena, Miguel Magallanes

\section{To cite this version:}

Manuel Tena, Miguel Magallanes. Relation entre l'activité fixatrice d'azote non symbiotique et quelques propriétés physicochimiques du sol. Agronomie, 1985, 5 (4), pp.369-373. hal-00884771

\section{HAL Id: hal-00884771 \\ https://hal.science/hal-00884771}

Submitted on 1 Jan 1985

HAL is a multi-disciplinary open access archive for the deposit and dissemination of scientific research documents, whether they are published or not. The documents may come from teaching and research institutions in France or abroad, or from public or private research centers.
L'archive ouverte pluridisciplinaire HAL, est destinée au dépôt et à la diffusion de documents scientifiques de niveau recherche, publiés ou non, émanant des établissements d'enseignement et de recherche français ou étrangers, des laboratoires publics ou privés. 


\title{
Relation entre l'activité fixatrice d'azote non symbiotique et quelques propriétés physico- chimiques du sol
}

\author{
Manuel TENA \& Miguel MAGALLANES \\ Cátedra de Bioquímica y Química Agrícola, Escuela Técnica Superior de Ingenieros Agrónomos, Universidad \\ de Coŕdoba, Apartado de Correos 3048, Córdoba, España
}

Mots clés additionnels : Corrélation, $\mathrm{pH}$, argile, potassium assimilable, azote total, azote ammoniacal.

Relationship between non-symbiotic nitrogen-fixing activity and some physicochemical soil properties.

Working with 47 soils, we have studied the relationship between nitrogen-fixing activity ( $\mathrm{N}_{2}$-ase activity) and some physicochemical soil properties. $\mathrm{N}_{2}$-ase activity was significantly correlated with $\mathrm{pH}\left(\mathrm{r}=0.72^{* * *}\right)$, clay $\left(\mathrm{r}=0.57^{* * *}\right)$, assimilable potassium $\left(\mathrm{r}=0.41^{* *}\right)$, total nitrogen $\left(\mathrm{r}=0.36^{*}\right)$ and ammonium nitrogen $\left(\mathrm{r}=-0.33^{*}\right)$. Multiple regression analyses showed that only $\mathrm{pH}$ and total and ammonium nitrogen significantly contributed to explaining in part the variation in $N_{2}$-ase activity $\left(R^{2}=58 \%\right)$.

Additional key words : Correlation, $p H$, clay, assimilable potassium, total nitrogen, ammonia nitrogen.

\section{INTRODUCTION}

L'étude des activités enzymatiques du sol est un thème fort intéressant actuellement, tant par leurs aspects fondamentaux que par la capacité potentielle que possède le sol pour réaliser d'importants processus biologiques relatifs à sa fertilité (BURNS, 1978).

La fixation biologique de l'azote atmosphérique est, dans le cycle de cet élément, un processus important qui se réalise grâce à un nombre réduit d'organismes procaryotes que l'on peut séparer du point de vue écologique en deux grands groupes : symbiotiques et non symbiotiques. Alors que l'importance de la fixation symbiotique de l'azote n'est plus mise en doute, nous ne pouvons pas dire la même chose pour la fixation non symbiotique de l'azote dont l'importance est un des sujets les plus discutés dans le domaine de la biochimie du sol. Cependant, on a mis en évidence que, dans certains cas, la fixation d'azote par des microorganismes non symbiotiques peut jouer un rôle important dans l'économie de l'azote de différents écosystèmes (DöBEREINER, 1968 ; YOSHIDA \& ANCAJAS, 1971, 1973a, $b$; DOMMERGUES et al., 1973 ; KLUBEK \& SKUJINS, 1980 ; CASSELMAN et al., 1981).

La bibliographie nous révèle que les sols, dans des conditions appropriées, montrent des valeurs appréciables d'activité fixatrice d'azote variant notablement selon les sols. Cependant il n'existe pratiquement aucune information sur la possibilité d'une relation entre cette activité et les propriétés physicochimiques du sol, et c'est sur cet aspect particulier que l'on a basé l'étude exposée dans ce travail. 


\section{MATÉRIEL ET MÉTHODES}

L'étude effectuée porte sur 47 sols cultivés provenant de diverses régions d'Andalousie occidentale (Espagne). Les échantillons sont prélevés dans la couche superficielle du sol $(0-15 \mathrm{~cm})$ et, avant d'être utilisés, ils sont séchés à l'air et tamisés à $2 \mathrm{~mm}$. Les caractéristiques physicochimiques des sols et les références des méthodes d'analyses utilisées figurent dans le tableau 1. Les taux d'azote ammoniacal $\left(\mathrm{N}-\mathrm{NH}_{4}\right)$ et d'azote inorganique total $\left(\mathrm{N}_{\mathrm{i}}-\mathrm{t}\right)$ sont déterminés dans les extraits obtenus en agitant les échantillons durant
$1 \mathrm{~h}$ avec une solution $2 \mathrm{~N}$ de $\mathrm{KCl}$ dans, la proportion $(\mathrm{p} / \mathrm{v})$ de $1: 10$.

L'activité fixatrice d'azote (activité $\mathrm{N}_{2}$-ase) est mesurée par la méthode de réduction d'acétylène (HARDY $e t$ al., 1973, PostGATE, 1971). On introduit des échantillons de $10 \mathrm{~g}$ de sol dans des petits flacons de $50 \mathrm{ml}$ additionnés de $0,1 \mathrm{~g}$ de glucose et de la quantité d'eau nécessaire pour obtenir différentes valeurs d'humidité, entre 60 et 150 p. 100 de la capacité de rétention d'eau (CRE). Les échantillons sont incubés pendant des temps divers, à $30^{\circ} \mathrm{C}$, à l'obscurité. Les flacons sont alors fermés avec un bouchon en caoutchouc avec une

TABLEAU 1

Composition, caractéristiques et activité $\mathrm{N}_{2}$-ase maximum des sols.

Composition, characteristics and maximum $\mathrm{N}_{2}$-ase activity of the soils.

\begin{tabular}{|c|c|c|c|c|c|c|c|c|c|}
\hline Sol & $\begin{array}{c}\mathrm{pH} \\
\left(\mathrm{H}_{2} \mathrm{O} \quad 1: 2,5\right)\end{array}$ & M.O. & $N_{1}$ & Argile & $\mathrm{N}-\mathrm{NH}_{4}$ & $\mathrm{~N}_{\mathrm{i}}-\mathrm{t}$ & $\mathbf{P}$ & $\mathbf{K}$ & Activité $\mathrm{N}_{2}$-ase maximum* \\
\hline & & & $\%$ & \multicolumn{3}{|c|}{$\% \times 10^{-3}$} & \multicolumn{2}{|c|}{ ppm } & \\
\hline 1 & 5,0 & 0,72 & 0,069 & 21,6 & 3,75 & 4,32 & 3,3 & 180 & $50,2 \pm 3,7(2)^{* *}$ \\
\hline 2 & 5,0 & 1,96 & 0,111 & 24,0 & 4,60 & 5,18 & 6,9 & 60 & $77,8 \pm 18,1(2)$ \\
\hline 3 & 5,1 & 1,20 & 0,072 & 10,0 & 4,10 & 5,78 & 6,0 & 40 & $44,4 \pm 7,8(2)$ \\
\hline 4 & 5,2 & 0,40 & 0,044 & 28,6 & 3,60 & 6,28 & 3,5 & 100 & $25,0 \pm 3,1(3)$ \\
\hline 5 & 5,2 & 0,50 & 0,052 & 32,4 & 6,00 & 6,64 & 18,3 & 135 & $26,8 \pm 2,4(3)$ \\
\hline 6 & 5,4 & 0,46 & 0,046 & 13,5 & 3,85 & 4,57 & 1,0 & 35 & $12,6 \pm 0,6(2)$ \\
\hline 7 & 5,4 & 1,08 & 0,071 & 13,5 & 4,25 & 4,46 & 2,1 & 31 & $21,2 \pm 3,1(1)$ \\
\hline 8 & 5,4 & 1,50 & 0,112 & 15,7 & 5,78 & 6,53 & 24,2 & 93 & $24,5 \pm 3,2(3)$ \\
\hline 9 & 5,5 & 0,44 & 0,031 & 10,0 & 4,39 & 6,78 & 2,1 & 23 & $8,8 \pm 3,9(3)$ \\
\hline 10 & 5,5 & 0,57 & 0,061 & 24,1 & 4,43 & 5,25 & 15,9 & 105 & $28,5 \pm 1,6(3)$ \\
\hline 11 & 5,5 & 1,04 & 0,068 & 24,6 & 4,35 & 5,03 & 3,2 & 30 & $30,3 \pm 4,2(3)$ \\
\hline 12 & 5,5 & 2,08 & 0,108 & 25,7 & 3,32 & 4,46 & 5,1 & 70 & $55,9 \pm 3,7(2)$ \\
\hline 13 & 5,6 & 0,50 & 0,054 & 23,0 & 2,89 & 4,21 & 9,0 & 70 & $43,9 \pm 5,8(2)$ \\
\hline 14 & 5,7 & 2,54 & 0,105 & 23,2 & 4,85 & 5,89 & 14,1 & 86 & $54,7 \pm 5,9(3)$ \\
\hline 15 & 5,8 & 3,00 & 0,137 & 13,2 & 5,21 & 5,21 & 15,6 & 109 & $44,4 \pm 4,6(1)$ \\
\hline 16 & 6,1 & 1,45 & 0,084 & 16,0 & 3,75 & 5,71 & 4,6 & 40 & $238,4 \pm 14,2(2)$ \\
\hline 17 & 6,5 & 0,83 & 0,060 & 13,3 & 4,18 & 4,39 & 2,0 & 125 & $169,7 \pm 18,1(2)$ \\
\hline 18 & 6,5 & 2,02 & 0,135 & 17,6 & 4,14 & 8,25 & 16,0 & 95 & $105,2 \pm 13,7(1)$ \\
\hline 19 & 6,7 & 0,94 & 0,076 & 20,1 & 3,32 & 4,14 & 7,5 & 113 & $178,4 \pm 29,6(2)$ \\
\hline 20 & 6,8 & 0,93 & 0,063 & 4,6 & 3,43 & 4,35 & 3,0 & 90 & $33,7 \pm 3,8(1)$ \\
\hline 21 & 7,0 & 0,67 & 0,038 & 19,8 & 3,75 & 6,78 & 8,0 & 320 & $66,9 \pm 16,3(2)$ \\
\hline 22 & 7,6 & 0,57 & 0,068 & 22,0 & 2,64 & 4,25 & 3,0 & 80 & $43,9 \pm 4,3(1)$ \\
\hline 23 & 7,7 & 0,67 & 0,057 & 33,3 & 4,10 & 12,70 & 23,0 & 290 & $38,9 \pm 1,4(3)$ \\
\hline 24 & 7,8 & 0,93 & 0,051 & 23,1 & 3,57 & 5,10 & 30,0 & 170 & $246,2 \pm 11,6(2)$ \\
\hline 25 & 7,8 & 1,04 & 0,087 & 7,3 & 4,18 & 4,28 & 19,0 & 80 & $101,4 \pm 15,6(2)$ \\
\hline 26 & 7,8 & 1,08 & 0,091 & 58,0 & 6,78 & 9,10 & 16,0 & 785 & $134,2 \pm 42,2(3)$ \\
\hline 27 & 7,8 & 1,22 & 0,094 & 62,8 & 2,50 & 5,10 & 7,0 & 295 & $341,0 \pm 18,2(2)$ \\
\hline 28 & 7,8 & 1,96 & 0,131 & 82,4 & 4,32 & 5,82 & 6,5 & 175 & $179,6 \pm 13,0(1)$ \\
\hline 29 & 7,9 & 1,03 & 0,088 & 65,3 & 3,03 & 4,10 & 6,0 & 280 & $266,1 \pm 8,1(2)$ \\
\hline 30 & 7,9 & 1,09 & 0,094 & 63,6 & 3,14 & 4,50 & 7,0 & 345 & $308,4 \pm 19,1(2)$ \\
\hline 31 & 7,9 & 1,19 & 0,104 & 43,4 & 4,75 & 5,71 & 15,0 & 208 & $186,1 \pm 46,8(1)$ \\
\hline 32 & 7,9 & 1,34 & 0,094 & 67,4 & 3,53 & 5,03 & 8,0 & 380 & $314,6 \pm 12,0(2)$ \\
\hline 33 & 8,0 & 0,97 & 0,084 & 60,8 & 3,39 & 3,82 & 4,5 & 165 & $150,6 \pm 15,5(2)$ \\
\hline 34 & 8,0 & 0,97 & 0,084 & 64,5 & 2,89 & 3,39 & 6,0 & 230 & $146,4 \pm 17,4(2)$ \\
\hline 35 & 8,0 & 1,03 & 0,090 & 64,2 & 3,03 & 4,25 & 6,5 & 290 & $209,0 \pm 27,2(2)$ \\
\hline 36 & 8,0 & 1,04 & 0,092 & 67,0 & 4,32 & 5,14 & 7,0 & 340 & $216,5 \pm 25,3(2)$ \\
\hline 37 & 8,0 & 1,09 & 0,090 & 62,4 & 4,75 & 5,03 & 6,5 & 240 & $185,9 \pm 32,7(1)$ \\
\hline 38 & 8,0 & 1,09 & 0,094 & 63,0 & 4,78 & 4,93 & 7,0 & 325 & $173,6 \pm 12,5(2)$ \\
\hline 39 & 8,1 & 0,88 & 0,069 & 45,7 & 3,28 & 3,89 & 1,5 & 105 & $106,7 \pm 13,7(3)$ \\
\hline 40 & 8,1 & 0,91 & 0,092 & 61,8 & 3,89 & 4,89 & 7,0 & 320 & $214,5 \pm 11,1(2)$ \\
\hline 41 & 8,1 & 0,94 & 0,081 & 41,2 & 3,03 & 3,53 & 10,5 & 155 & $191,1 \pm 16,5(1)$ \\
\hline 42 & 8,1 & 1,14 & 0,082 & 25,9 & 4,39 & 5,89 & 14,0 & 242 & $346,7 \pm 18,2(1)$ \\
\hline 43 & 8,1 & 1,30 & 0,090 & 52,0 & 4,96 & 5,68 & 13,2 & 136 & $112,3 \pm 18,1(2)$ \\
\hline 44 & 8,2 & 1,50 & 0,103 & 76,3 & 3,57 & 3,57 & 3,0 & 80 & $191,0 \pm 53,6(1)$ \\
\hline 45 & 8,3 & 0,73 & 0,084 & 63,5 & 2,43 & 3,43 & 3,0 & 160 & $207,6 \pm 52,8(3)$ \\
\hline 46 & 8,3 & 1,34 & 0,105 & 63,4 & 5,43 & 5,43 & 11,0 & 999 & $166,6 \pm 42,2(3)$ \\
\hline 47 & 8,3 & 1,82 & 0,126 & 24,7 & 2,78 & 4,93 & 43,0 & 301 & $313,1 \pm 31,1(1)$ \\
\hline
\end{tabular}

*n moles $\mathrm{C}_{2} \mathrm{H}_{4} \cdot \mathrm{g}^{-1}$ sol. h. ${ }^{-1}$.

** Les données entre parenthèses, indiquent le temps d'incubation avec du glucose (exprimé en jours) nécessaire pour le développement de l'activité $\mathrm{N}_{2}$-ase maximum.

M.O. : Walkley \& Black (1934); N : Bremner (1965); Argile : Day (1965) ; P : Olsen \& Dean (1965) ; K : Pratt (1965). 
coiffe métallique ; on injecte ensuite $5 \mathrm{ml}$ d'acétylène. Après $24 \mathrm{~h}$, on mesure l'éthylène produit par chromatographie en phase gazeuse avec un appareil équipé d'un détecteur à ionisation de flamme (Perkin-Elmer mod. 3920B) muni d'une colonne en acier inoxydable de $200 \times 0,32 \mathrm{~cm}$ remplie de Porapak $\mathrm{N}$ de 80 à 100 mesh. On utilise l'azote comme gaz vecteur avec un débit d'environ $40 \mathrm{ml} \cdot \mathrm{min}^{-1}$. Les températures de l'injecteur, de la colonne et du détecteur, sont respectivement de 155,70 et $200{ }^{\circ} \mathrm{C}$. Le volume de l'injection est de $1 \mathrm{ml}$. Les concentrations d'éthylène sont déterminées en injectant alternativement des échantillons et des standards et en comparant la surface des pics respectifs. Pour mesurer la surface des pics on utilise un intégrateur Hewlett-Packard mod. 3373B. On réalise tous les essais dans l'intervalle de réponse linéaire du détecteur. On exprime l'activité $\mathrm{N}_{2}$-ase en nmol $\mathrm{C}_{2} \mathrm{H}_{4} \cdot \mathrm{g}^{-1}$ de sol.h $\mathrm{h}^{-1}$.

L'analyse statistique est effectuée au moyen d'un micro-ordinateur Hewlett-Packard mod. A-9380.

\section{RÉSULTATS}

Afin d'obtenir une estimation valable de l'activité fixatrice d'azote (activité $\mathrm{N}_{2}$-ase) des sols, on a étudié d'abord l'influence d'une série de facteurs (addition de glucose, humidité et temps d'incubation avec l'acétylène) sur la dite activité :

- Dans les sols non traités avec du glucose, on a détecté une activité $\mathrm{N}_{2}$-ase négligeable (résultats non exposés dans ce travail); cependant avec 1 p. 100 de glucose, les sols ont déployé une activité considérable.

- L'influence de l'humidité a été étudiée dans 2 types de sols en utilisant une humidité comprise entre 60 et 150 p. 100 de la CRE. Dans un des 2 sols (fig. 1A) on a obtenu une activité $\mathrm{N}_{2}$-ase maximum avec une humidité de 120 p. 100 de la CRE ; dans l'autre sol, on a obtenu l'activité maximum pour une humidité de 90 p. 100 de la CRE (fig. 1B) bien que, dans ce cas, on n'ait pas apprécié de différences notables entre 90 et 150 p. 100 d'humidité. Dans les 2 cas on a détecté une activité $\mathrm{N}_{2}$-ase négligeable avec 60 p. 100 d'humidité (résultats non exposés dans ce travail). En conséquence de ces études préliminaires, on a choisi une humidité de 120 p. 100 de la CRE considérée comme optimale pour la détermination de l'activité $\mathrm{N}_{2}$-ase des différents sols.

- Enfin, pour le temps d'incubation avec l'acétylène, l'étude réalisée avec un sol a démontré qu'après une incubation avec le glucose durant le temps nécessaire pour le développement de l'activité $\mathrm{N}_{2}$-ase maximum (voir ci-après), l'addition de l'acétylène conduisait à une production d'éthylène suivant une cinétique d'ordre zéro, au moins durant les $30 \mathrm{~h}$ suivantes (fig. 2). Selon ce résultat, et afin d'obtenir une réponse suffisante pour les essais effectués avec les différents sols, on a choisi $24 \mathrm{~h}$ comme temps d'incubation avec l'acétylène.

Les analyses de l'activité $\mathrm{N}_{2}$-ase dans les différents sols ont indiqué qu'elle augmentait jusqu'à une valeur maximum en même temps qu'augmentait le temps d'incubation avec le glucose avant l'addition d'acétylène. Le temps d'incubation nécessaire pour le développement de l'activité maximum a varié entre 1 et $3 \mathrm{j}$
Activité $\mathrm{N}_{2}$-ase ( $\left.\mathrm{nmol} \mathrm{C} 2 \mathrm{H}_{4} \mathrm{~g}^{-1} \mathrm{sol} h-1\right)$
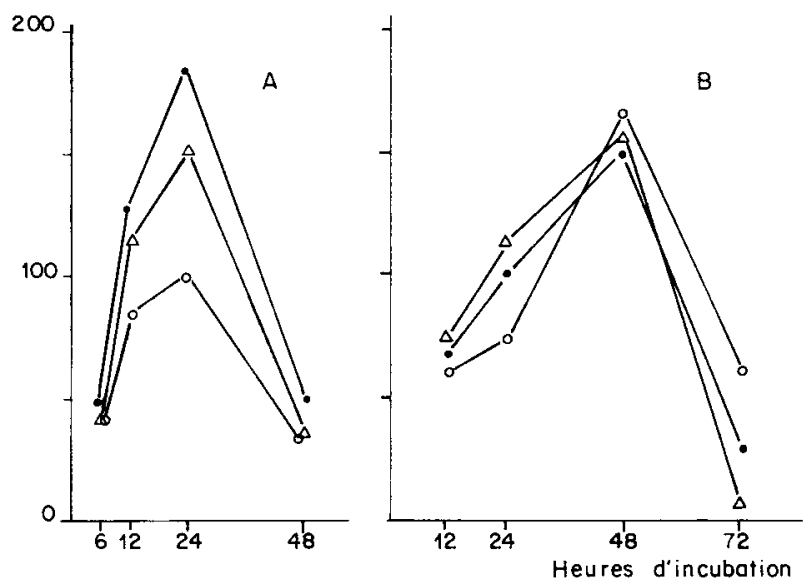

Figure 1

Relation entre l'activité $N_{2}$-ase et l'humidité du sol. Essais réalisés avec les sols $n^{\circ} 28(A)$ et $33(B)$. Les échantillons sont incubés avec du glucose. Après les temps indiqués, on injecte l'acétylène et on mesure l'éthylène produit après $24 \mathrm{~h}$.

O 90 p. 100 C.R.E.

- 120 p. 100 C.R.E

$\triangle 150$ p. 100 C.R.E.

Relation between $\mathrm{N}_{2}$-ase activity and soil moisture. Assays performed with soils $28(A)$ and 33 (B). Soil samples were incubated with glucose. At the intervals indicated acetylene was injected and the ethylene produced after $24 h$ was measured.

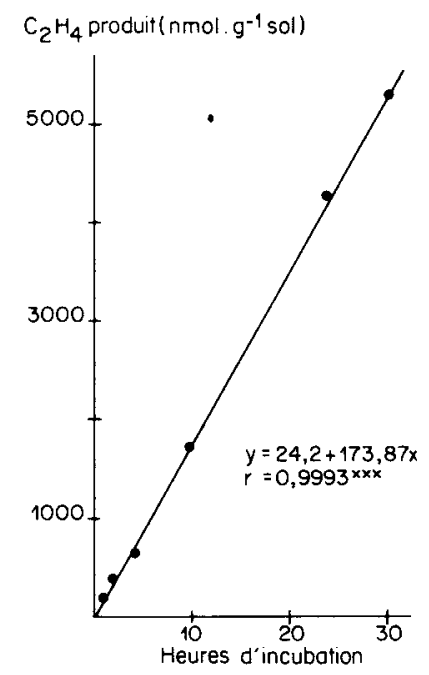

Figure 2

Ethylène produit à différents temps d'incubation avec l'acétylène. Essai réalisé avec le sol $n^{\circ} 28$. Les échantillons sont incubés $24 h$ avec du glucose, ensuite on injecte l'acétylène et on mesure l'éthylène produit après les intervalles indiqués.

Ethylene produced at differents times of incubation with acetylene. Assay performed with soil 28. Soil samples were incubated $24 \mathrm{~h}$ with glucose, then acetylene was injected and, at the intervals indicated, the ethylene produced was measured.

selon les sols (fig. 3). L'activité $\mathrm{N}_{2}$-ase maximum fut bien différente selon les sols. Ainsi (voir fig. 3), dans 19 des 47 sols étudiés, l'activité maximum fut inférieure à $100 \mathrm{nmol} \mathrm{C}_{2} \mathrm{H}_{4} \cdot \mathrm{g}^{-1}$ de sol.h-1, et dans 16 , 7 et 5 sols, elle fut respectivement comprise entre 100 200, 200-300 et 300-400 nmol $\mathrm{C}_{2} \mathrm{H}_{4} \cdot \mathrm{g}^{-1}$ de sol.h ${ }^{-1}$. Les valeurs de l'activité $\mathrm{N}_{2}$-ase maximum des sols et les temps d'incubation avec le glucose, avant l'addi- 


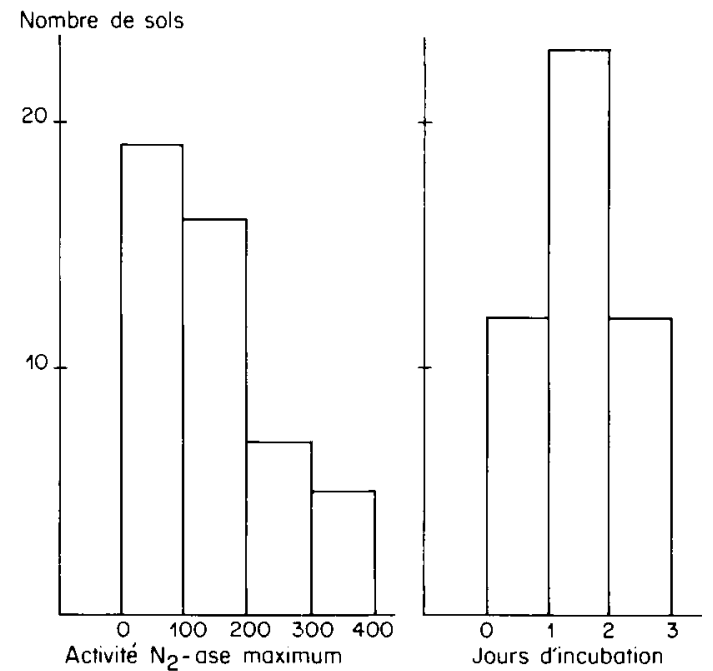

Figure 3

A. Activité $\mathrm{N}_{2}$-ase maximum des sols. Maximum $\mathrm{N}_{2}$-ase activity of the soils.

B. Temps d'incubation avec glucose pour obtenir l'activité $\mathrm{N}_{2}$-ase maximum.

Time of incubation with glucose to obtain the maximum $\mathrm{N}_{2}$-ase activity.

tion d'acétylène, nécessaires pour obtenir les dites valeurs figurent dans le tableau 1.

Comme on peut le voir dans le tableau 2, l'activité $\mathrm{N}_{2}$-ase maximum des sols est fortement corrélée $(\mathrm{p}<0,001)$ à leur $\mathrm{pH}$ et à leur teneur en argile, ainsi qu'à leurs taux de potassium assimilable $(p<0,01)$ et d'azotes total et ammoniacal $(p<0,05)$. Toutes ces corrélations sont positives à l'exception de celle avec l'azote ammoniacal. Cependant, malgré l'existence d'une forte corrélation entre l'azote total et la matière organique $\left(\mathrm{r}=0,84^{* * *}\right)$, l'activité $\mathrm{N}_{2}$-ase maximum n'est pas corrélée significativement avec la matière organique.

L'analyse en régression linéaire multiple nous révèle que les teneurs en argile et en potassium assimilable n'ont aucune influence significative sur la variabilité

\section{TABLEAU 2}

Coefficients de corrélation entre l'activité $\mathrm{N}_{2}$-ase maximum et d'autres propriétés du sol.

Correlation coefficients between maximum $\mathrm{N}_{2}$-ase activity and other soil properties.

\begin{tabular}{lc}
\hline \multicolumn{1}{c}{ Propriétés du sol } & $\begin{array}{c}\text { Coefficients } \\
\text { de corrélation (r) }\end{array}$ \\
\hline Matière Organique (M.O) & 0,12 \\
Azote total $\left(\mathrm{N}_{1}\right)$ & $0,36^{*}$ \\
Relation $\mathrm{M} . \mathrm{O} / \mathrm{N}_{\mathrm{t}}$ & $-0,07$ \\
Azote ammoniacal $\left(\mathrm{N}-\mathrm{NH}_{4}\right)$ & $-0,33^{*}$ \\
Azote inorganique total $\left(\mathrm{N}_{\mathrm{i}} \mathrm{t}\right)$ & $-0,23$ \\
Relation M.O/ $/ \mathrm{N}_{\mathrm{i}}-\mathrm{t}$ & 0,21 \\
Phosphore assimilable & 0,16 \\
Potassium assimilable & $0,41^{* *}$ \\
Argile & $0,57^{* * *}$ \\
$\mathrm{pH}$ & $0,72^{* * *}$ \\
\hline \hline
\end{tabular}

$\left(^{*}\right)$ P 0,$05 ;\left({ }^{* *}\right)$ P 0,$01 ;\left({ }^{* * *}\right)$ P 0,001 .
TABLEAU 3

Coefficients numériques (valeurs de b) des propriétés du sol dans l'équation de corrélation (1) et sa signification.

Numerical coefficients (b-values) of soil properties in equation (l) and significance.

\begin{tabular}{lc}
\hline \hline \multicolumn{1}{c}{ Propriétés du sol } & $\begin{array}{c}\text { Coefficients } \\
\text { numériques }\end{array}$ \\
\hline Intersection & $-193,15$ \\
Azote total $\left(\mathrm{N}_{4}\right)$ & $893,68^{*}$ \\
Azote ammoniacal $\left(\mathrm{N}-\mathrm{NH}_{4}\right)$ & $-22,30^{*}$ \\
pH & $49,36^{* * *}$ \\
\hline
\end{tabular}

$\left(^{*}\right)$ P 0,$05 ;\left(^{* *}\right)$ P 0,$01 ;\left({ }^{* * *}\right)$ P 0,001 .

de l'activité $\mathrm{N}_{2}$-ase maximum ; on a obtenu l'équation de régression suivante :

$$
\begin{gathered}
\text { [1] activité } \quad \mathrm{N}_{2} \text {-ase }=-193,2+893,7 \quad\left(\mathrm{~N}_{t}\right)-22,3 \\
\left(\mathrm{~N}_{\mathrm{N}} \mathrm{NH}_{4}\right)+49,4(\mathrm{pH}) \\
\mathrm{R}^{2}=58 \% .
\end{gathered}
$$

Les significations des coefficients numériques de cette équation se trouvent dans le tableau 3.

\section{DISCUSSION ET CONCLUSIONS}

Les résultats obtenus dans l'étude préliminaire sur les conditions optima des essais de l'activité $\mathrm{N}_{2}$-ase sont en général conformes avec les données de la bibliographie. Ainsi, la nécessité, pour le développement de l'activité $\mathrm{N}_{2}$-ase du sol, d'une source de carbone facilement utilisable a été constatée par divers auteurs (KNOWLES, 1976, et références citées dans ce travail). De même, l'effet favorable d'une forte humidité est en accord avec le fait connu qu'en présence de matières organiques il se développe une activité $\mathrm{N}_{2}$-ase plus intense dans les sols incubés en anaérobiose que dans ceux incubés en aérobiose, ou bien que, lorsqu'il existe des conditions aérobies, l'engorgement des sols augmente leur capacité $\mathrm{N}_{2}$-ase (KNOWLES, 1976 ; RAO, 1976). Ce fait a également été constaté en présence de certains microorganismes aérobies comme Azotobacter, ce qui paraît indiquer qu'avec des conditions aérobies il existe une plus forte compétition entre les hétérotrophes fixateurs et les non fixateurs de l'azote pour accaparer les sources de carbone organique (KNOWLES, 1976).

Nos résultats montrent qu'avec des conditions optima d'incubation, il existe une différence notable entre les activités $\mathrm{N}_{2}$-ase maximum des divers sols, qui peut être due, au moins partiellement, aux propriétés physicochimiques du sol. Ainsi l'activité $\mathrm{N}_{2}$-ase est fortement corrélée au pH et à la teneur en argile; de même, à un moindre degré de signification, aux taux en potassium assimilable, azote total et azote ammoniacal (tabl. 2). D'autre part, l'équation de régression [1] met en évidence que plus de la moitié (environ 60 p. 100) de la variation de l'activité $\mathrm{N}_{2}$-ase des sols peut être expliquée par le $\mathrm{pH}$ et les teneurs en azotes total et ammoniacal du sol. Le coefficient très significatif du $\mathrm{pH}$ dans l'équation de régression multiple 
montre que cette propriété du sol est celle qui apparemment a le plus grand effet sur l'activité $\mathrm{N}_{2}$-ase (tabl. 3). Ce résultat est en accord avec les résultats des études de corrélation simple (tabl. 2). A notre connaissance l'existence d'une telle relation entre le $\mathrm{pH}$ et l'activité $\mathrm{N}_{2}$-ase du sol n'avait pas encore été établie, bien qu'elle ait été ébauchée dans diverses études réalisées avec un nombre limité de sols (YONEYAMA et al., 1977 ; CHARYULU \& RAO, 1981) ou avec des cultures enrichies en organismes fixateurs d'azote provenant de différents sols (DöBEREINER et al., 1976 ; CHARYULU \& RAO, 1980). Nos résultats indiquent aussi l'existence d'une relation inverse entre l'activité $\mathrm{N}_{2}$-ase et la teneur en azote ammoniacal du sol (tabl. 2, équation [1]). Plusieurs travaux ont conclu que l'addition d'ammonium au sol inhibe fortement son activité $\mathrm{N}_{2}$-ase (KNOWLES \& DENIKE, 1974 ; RAO, 1976 ; CHAR YULU \& RAO, 1981) et ont établi en outre qu'en cultures pures, l'ammonium est un répresseur de l'enzyme nitrogénase (DALTON \& MORTENSON, 1972). Cependant, l'effet de la teneur en ammonium du sol, sur l'activité $\mathrm{N}_{2}$-ase n'a pas suscité d'intérêt. Concrètement, on n'a trouvé qu'un seul travail dans la bibliographie arrivant à la conclusion d'une relation inverse entre l'activité $\mathrm{N}_{2}$-ase et la teneur en ammonium dans les sols marécageux (CASSELMAN et al., 1981). Nos résultats confirment et généralisent cette conclusion.

Recu le 24 avril 1984. Accepté le 4 décembre 1984.

\section{REMERCIEMENTS}

Ce travail a été financé par la "Comisión Asesora de Investigación Cientifica y Técnica » (projet de recherche $n^{\circ} 3826$ C02-01).

\section{RÉFÉRENCES BIBLIOGRAPHIQUES}

Bremner J. M., 1965. Total nitrogen, inorganic forms of nitrogen, and organic forms of nitrogen, p. 1149-1255. In C. A. Black : «Methods of soil analysis, Part 2 ». ASA, Madison, Wisc.

Burns R. G., 1978. Soil enzymes. Academic Press, London, 380 p. Casselman M. E., Patrick W. H., Delaune R. D., 1981. Nitrogen fixation in a gulf coast salt marsh. Soil Sci. Soc. Am. J., 45, 51-56. Charyulu P. B. B. N., Rao V. R., 1980. Influence of various soil factors on nitrogen fixation by Azospirillum spp. Soil Biol. Biochem., 12, 343-346.

Charyulu P. B. B. N., Rao V. R., 1981. Influence of ammonium nitrogen on nitrogen fixation in paddy soils. Soil Sci., 131, 140-144.

Dalton H., Mortenson L. E., 1972. Dinitrogen $\left(\mathrm{N}_{2}\right)$ fixation (with a biochemical emphasis). Bact. Rev., 36, 231-260.

Day P. R., 1965. Particle fractionation and particle-size analysis, p. 545-567. In C. A. Black : "Methods of soil analysis, Part 1 ». ASA, Madison, Wisc.

Döbereiner J., 1968. Non-symbiotic nitrogen fixation in tropical soils. Pesq. Agropec. Bras., 3, 1-6.

Döbereiner J., Marriel I. E., Nery M., 1976. Ecological distribution of Spirillum lipoferum Beijerinck. Can. J. Microbiol., 22, 1464-1463.

Dommergues Y., Balandreau J., Rinaudo G., Weinhard P., 1973. Non-symbiotic nitrogen fixation in the rhizospheres of rice, maize and different tropical grasses. Soil Biol. Biochem., 5, 83-89.

Hardy R. W. F., Burns R. C., Holstein R. D., 1973. Applications of the acetylene-ethylene assay for measurement of nitrogen fixation. Soil Biol. Biochem., 5, 47-81.

Klubek B., Skujins J., 1980. Heterotrophic $\mathrm{N}_{2}$-fixation in arid soil crusts. Soil Biol. Biochem., 12, 229-236.
Knowles R., 1976. Factors affecting dinitrogen fixation by bacteria in natural and agricultural systems. Proc. Ist Int. Symp. Nitrogen Fixation, vol. 2, p. 539-555. Washington State Univ. Press. Pullman, Wash.

Knowles R., Denike D., 1974. Effect of ammonium, nitrite and nitrate nitrogen on anaerobic nitrogenase activity in soil. Soil Biol. Biochem., 6, 353-358.

Olsen S. R., Dean L. A., 1965. Phosphorus, p. 1035-1049. In C. A. Black : "Methods of soil analysis, Part 2 ". ASA, Madison, Wisc.

Pratt P. F., 1965. Potassium, p. 1022-1030. In C. A. Black: «Methods of soil analysis, Part 2 ». ASA, Madison, Wisc.

Postgate J. R., 1971. The acetylene reduction test for nitrogen fixation. Methods Microbiol., 6B, 343-356.

Rao V. R., 1976. Nitrogen fixation as influenced by moisture content, ammonium sulphate and organic sources in a paddy soil. Soil Biol. Biochem., 8, 445-448.

Walkley A., Black I. A., 1934. An examination of the Degtjareff method for determining soil organic matter and a proposed modification of the chromic titration method. Soil Sci., 37, 29-38.

Yoneyama T., Lee K. K., Yoshida T., 1977. Decomposition of rice residues in tropical soils. IV. The effect of rice straw on nitrogen fixation by heterotrophic bacteria in some Philippine soils. Soil Sci. Plant Nutr., 23, 287-295.

Yoshida T., Ancajas R. R., 1971. Nitrogen fixation by bacteria in the root zone of rice. Soil Sci. Soc. Am. Proc., 35, 156-158.

Yoshida T., Ancajas R. R., 1973a. Nitrogen-fixing activity in upland and flooded rice fields. Soil Sci. Soc. Am. Proc., 37, 42-46.

Yoshida T., Ancajas R. R., 1973 $b$. The fixation of atmospheric nitrogen in the rice rhizosphere. Soil Biol. Biochem., 5, 153-155. 